Supporting Information

\title{
Discovery of Novel Small Molecule Inhibitors Disrupting the PCSK9-LDLR Interaction
}

\author{
Hengzhi Sun, ${ }^{\dagger \S}$ Jinzheng Wang,${ }^{\dagger \S}$ Shengjie Liu, ${ }^{\dagger \S}$ Xinyu Zhou,${ }^{\dagger}$ Liang Dai, ${ }^{\dagger}$ Caiping \\ Chen, ${ }^{\dagger}$ Qinglong Xu,${ }^{\dagger}$ Xiaoan Wen,${ }^{\dagger}$ Keguang Cheng, ${ }^{\dagger}$ Hongbin Sun,${ }^{\dagger *}$ and Haoliang \\ Yuan ${ }^{\dagger *}$ \\ $\dagger$ Jiangsu Key Laboratory of Drug Discovery for Metabolic Disease and State Key \\ Laboratory of Natural Medicines, China Pharmaceutical University, Nanjing 210009, \\ P.R. China \\ \$ State Key Laboratory for Chemistry and Molecular Engineering of Medicinal \\ Resources, School of Chemistry and Pharmacy, Guangxi Normal University, 15 Yucai \\ Road, Guilin 541004, P.R. China
}

\footnotetext{
*Corresponding Author.

*Email: hongbinsun@cpu.edu.cn (H. S.). Tel/Fax: +86-25-83271050.

*Email: yhl@cpu.edu.cn (H.Y.). Tel/Fax: +86-25-83271050.
}

\section{Notes}

The authors declare no competing financial interest.

\section{Author Contributions}

${ }^{\S}$ H.S., J.W. and S.L. contributed equally to this work.

Title Running Head: PCSK9-LDLR PPI; Small Molecule Inhibitors; Virtual Screening; Molecular Dynamics Simulation; Hypercholesterolemia 


\section{Table of Contents}

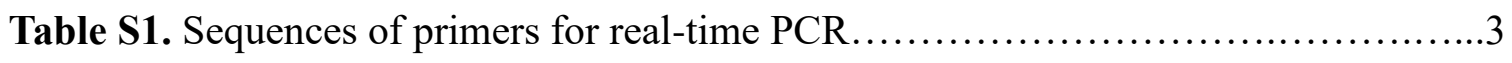

Table S2. The Specs ID numbers and chemical structures of all the 30 purchased compounds...............................................................4

Table S3. The preliminary inhibitory rates of selected compounds against PCSK9-LDLR

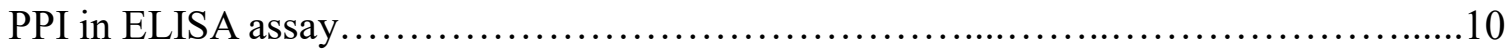

Figure S1. The inhibitory activities of $\mathbf{1 3}$ and pep2-8 against PCSK9-LDLR binding

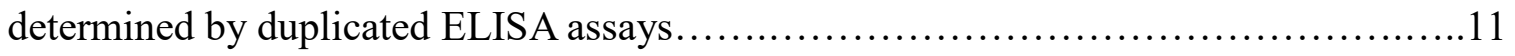

Figure S2. The mass spectrometry analysis of compound $13 \ldots \ldots \ldots \ldots \ldots \ldots \ldots \ldots \ldots \ldots \ldots \ldots$

Figure S3. The $K_{\mathrm{D}}$ values of $\mathbf{1 3}$ obtained from three independent SPR tests..............13

Figure S4. The RMSD values of the protein-ligand complex of PCSK9 and $\mathbf{1 3}$ in the

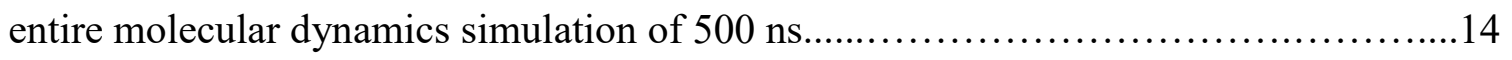

Figure S5. The effect of $\mathbf{1 3}$ on cell viability was determined with MTT assay...........15 
Table S1. Sequences of primers for real time PCR.

\begin{tabular}{cll}
\hline Gene & \multicolumn{1}{c}{ Forward Primer } & \multicolumn{1}{c}{ Reverse Primer } \\
\hline \multirow{2}{*}{ GAPDH } & 5'-GGAGCGAGATCCCTCCAA & 5'-GGCTGTTGTCATACTTCTC \\
& AAT-3' & ATGG-3' \\
\multirow{2}{*}{ LDLR } & 5'-TCTGCAACATGGCTAGAG & 5'-TCCAAGCATTCGTTGGTC \\
& ACT-3' & CC-3' \\
PCSK9 & 5'-AGACCCACCTCTCGCAGT & 5'-GGAGTCCTCCTCGATGTA \\
& C-3' & GTC-3' \\
\hline
\end{tabular}


Table S2. The Specs ID numbers and chemical structures of all the 30 purchased compounds.

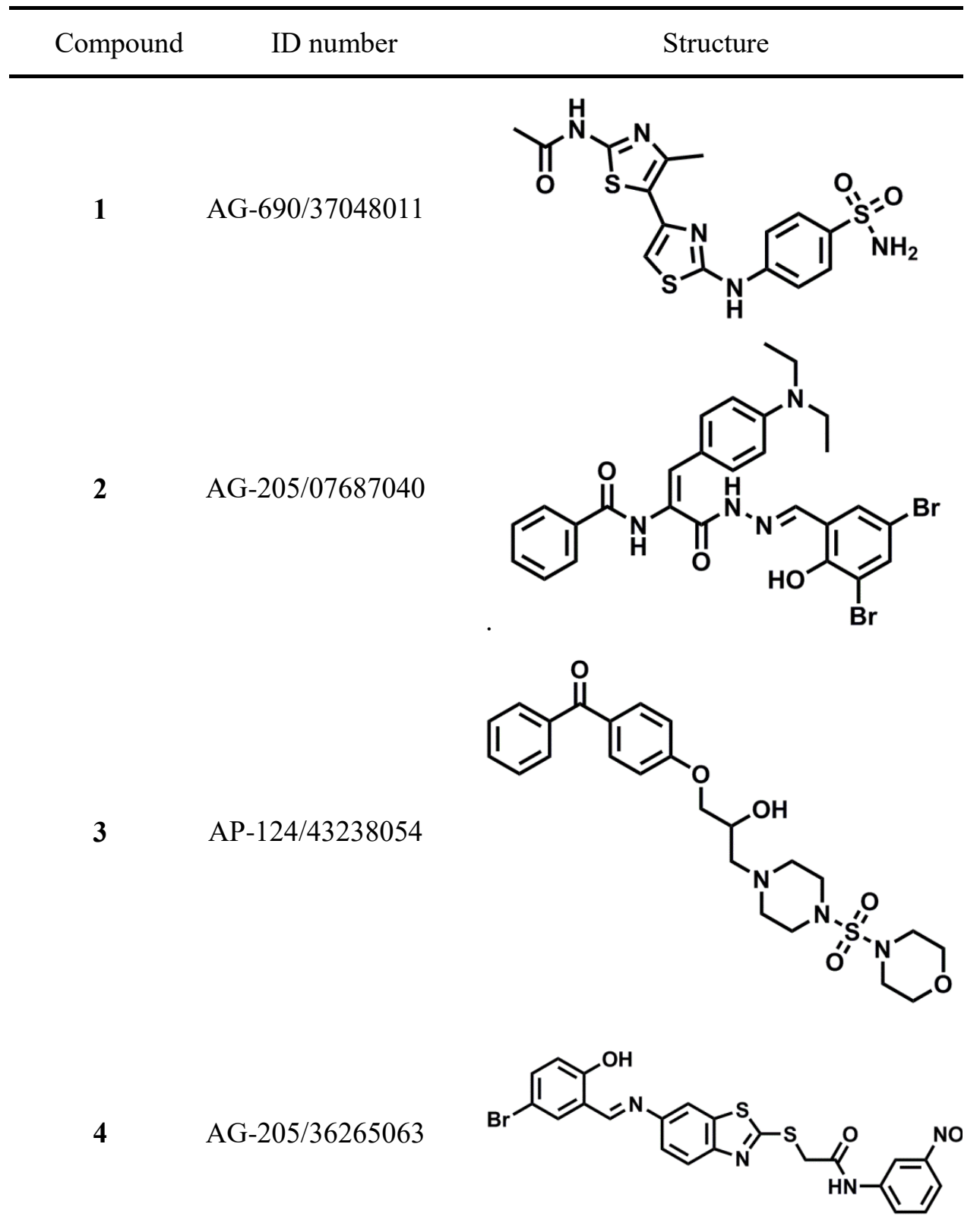


5

AG-690/33356049<smiles>O=C(O)c1ccc(C(=O)O)c(C(=O)Nc2ccc(NC(=O)c3cc(C(=O)O)ccc3C(=O)O)cc2)c1</smiles>

6

AN-153/13396332<smiles>COc1cccc(C(=O)Nc2ccc(NC(=O)c3ccco3)c(C)c2)c1</smiles>

7

AH-487/41802246<smiles>O=C(COc1ccc(S(=O)(=O)NC2CCCCC2)cc1)Nc1ccc(OCc2ccccc2)cc1</smiles>

8

AN-329/43448900<smiles>CC(C)C(=O)Nc1ccc(NC(=O)C2CCCCC2C(=O)O)cc1</smiles>

9

AN-329/43450396<smiles>CCOCCOc1ccc(C(=O)NCCc2ccccc2)cc1</smiles>

10

AK-968/13026003<smiles>COc1ccc(C(=O)Nc2cc(C(N)=O)cc(C(N)=O)c2)cc1</smiles> 


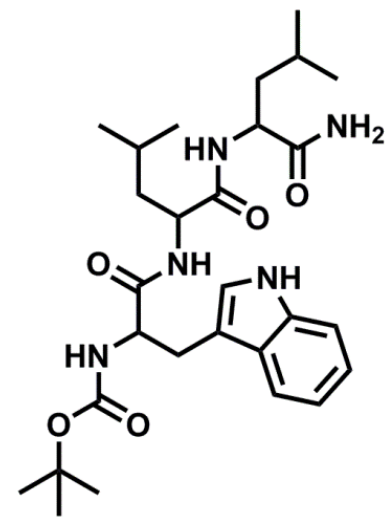

12

AN-988/15131258

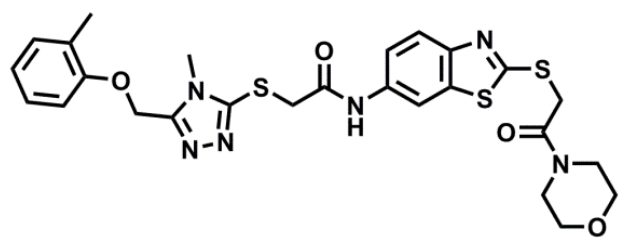

13

AN-919/15529007

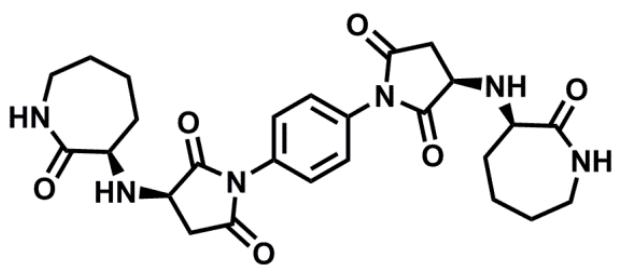

14

AG-690/11450136<smiles>CCN(CC)c1ccc(/C=N/NC(=O)c2cc(O)cc(O)c2)c(O)c1</smiles>

15

AJ-292/40711447<smiles>CCC(C)C(NC(=O)OC(C)(C)C)C(=O)NC(C(=O)NC(CCC(=O)OCc1ccccc1)C(N)=O)C(C)C</smiles> 


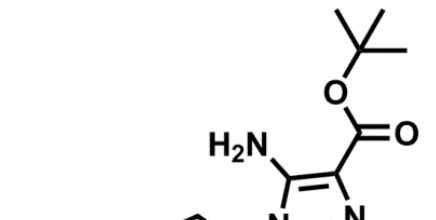

17

AJ-292/41694641<smiles>NS(=O)(=O)c1ccc(N2CN=NN2)cc1</smiles>
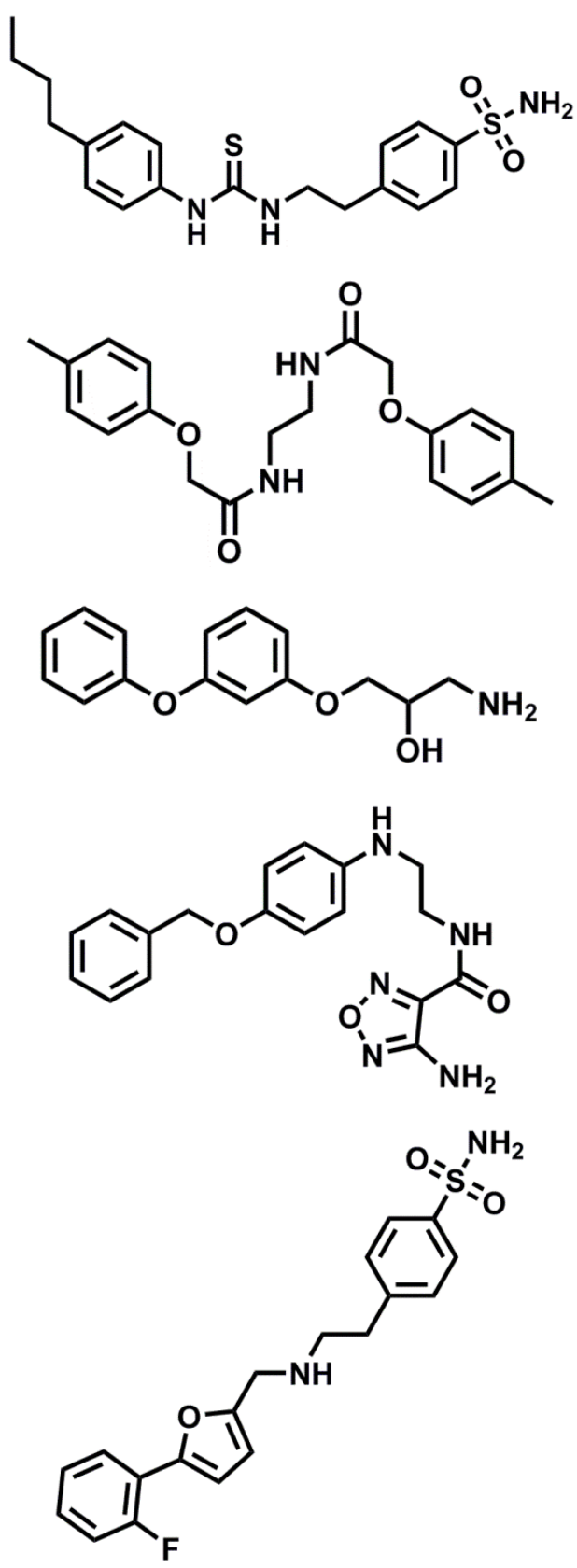
<smiles>O=C(NC(=S)Nc1ccccc1O)c1cccc(OCCOc2ccccc2)c1</smiles>

23

AO-080/43378361<smiles>CC(C)(c1ccccc1)c1ccc(OCCNC(=O)COCC(=O)O)cc1</smiles><smiles>CC(C)C(=O)Nc1ccc(C(=O)NCc2ccc(S(N)(=O)=O)cc2)cc1</smiles>

25

AO-476/43417722<smiles>CNC(=O)NC(=O)CSc1nnc(-c2ccc(C)cc2)n1N</smiles><smiles>COc1cccc(C(=O)Nc2ccc(NC(=O)c3cccs3)cc2)c1</smiles>

27 AK-968/41171874<smiles>COc1cc(NC(=O)CSCC(=O)O)cc(-n2cnnn2)c1</smiles> 


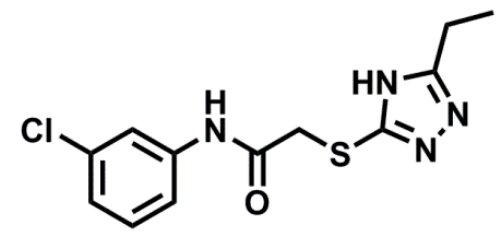

$29 \quad$ AN-465/41990292<smiles>CC(C)(C)NC(=O)COc1ccc(CNCCCN2CCOCC2)cc1</smiles>

30 AN-465/43411025<smiles>O=C(O)c1cccc(-c2ccc(CNCC(O)c3ccccc3)o2)c1</smiles> 
Table S3. The preliminary inhibitory rates of selected compounds against PCSK9-LDLR PPI in ELISA assay.

\begin{tabular}{ccc|ccc}
\hline compound $^{\mathrm{a}}$ & Inhibition Rate (\%) $^{\text {InSD }}$ & compound $^{\mathrm{a}}$ & Inhibition Rate (\%) $^{\mathbf{4}} \mathbf{\text { SD }}$ \\
\hline DMSO $_{\text {pep2-8 }}$ & 8.00 & 0.80 & $\mathbf{1 5}$ & -11.37 & 3.01 \\
$\mathbf{1}$ & 103.28 & 0.10 & $\mathbf{1 6}$ & -1.89 & 1.39 \\
$\mathbf{2}$ & 14.11 & 10.62 & $\mathbf{1 7}$ & -2.01 & 6.02 \\
$\mathbf{3}$ & 41.97 & 6.77 & $\mathbf{1 8}$ & -2.70 & 1.75 \\
$\mathbf{4}$ & 9.05 & 3.01 & $\mathbf{1 9}$ & 0.00 & 10.85 \\
$\mathbf{5}$ & 4.04 & 0.84 & $\mathbf{2 0}$ & -1.85 & 6.22 \\
$\mathbf{6}$ & -3.04 & 1.85 & $\mathbf{2 1}$ & -2.96 & 0.71 \\
$\mathbf{7}$ & 82.82 & 1.72 & $\mathbf{2 2}$ & -2.01 & 5.02 \\
$\mathbf{8}$ & 45.93 & 1.55 & $\mathbf{2 3}$ & -0.86 & 3.59 \\
$\mathbf{9}$ & 2.80 & 1.30 & $\mathbf{2 4}$ & 0.49 & 3.98 \\
$\mathbf{1 0}$ & 14.48 & 0.97 & $\mathbf{2 5}$ & 0.86 & 0.68 \\
$\mathbf{1 1}$ & -0.33 & 5.28 & $\mathbf{2 6}$ & 14.48 & 4.73 \\
$\mathbf{1 2}$ & 4.04 & 0.65 & $\mathbf{2 7}$ & -5.39 & 3.11 \\
$\mathbf{1 3}$ & 1.11 & 7.12 & $\mathbf{2 8}$ & -3.63 & 1.20 \\
$\mathbf{1 4}$ & 64.20 & 0.71 & $\mathbf{2 9}$ & 2.00 & 5.73 \\
\hline
\end{tabular}

${ }^{a}$ The concentration of selected compounds in the preliminary screening was set as $25 \mu \mathrm{M}$.

${ }^{\mathrm{b}}$ Pep2-8 at the concentration of $10 \mu \mathrm{M}$ was applied as the positive control. 

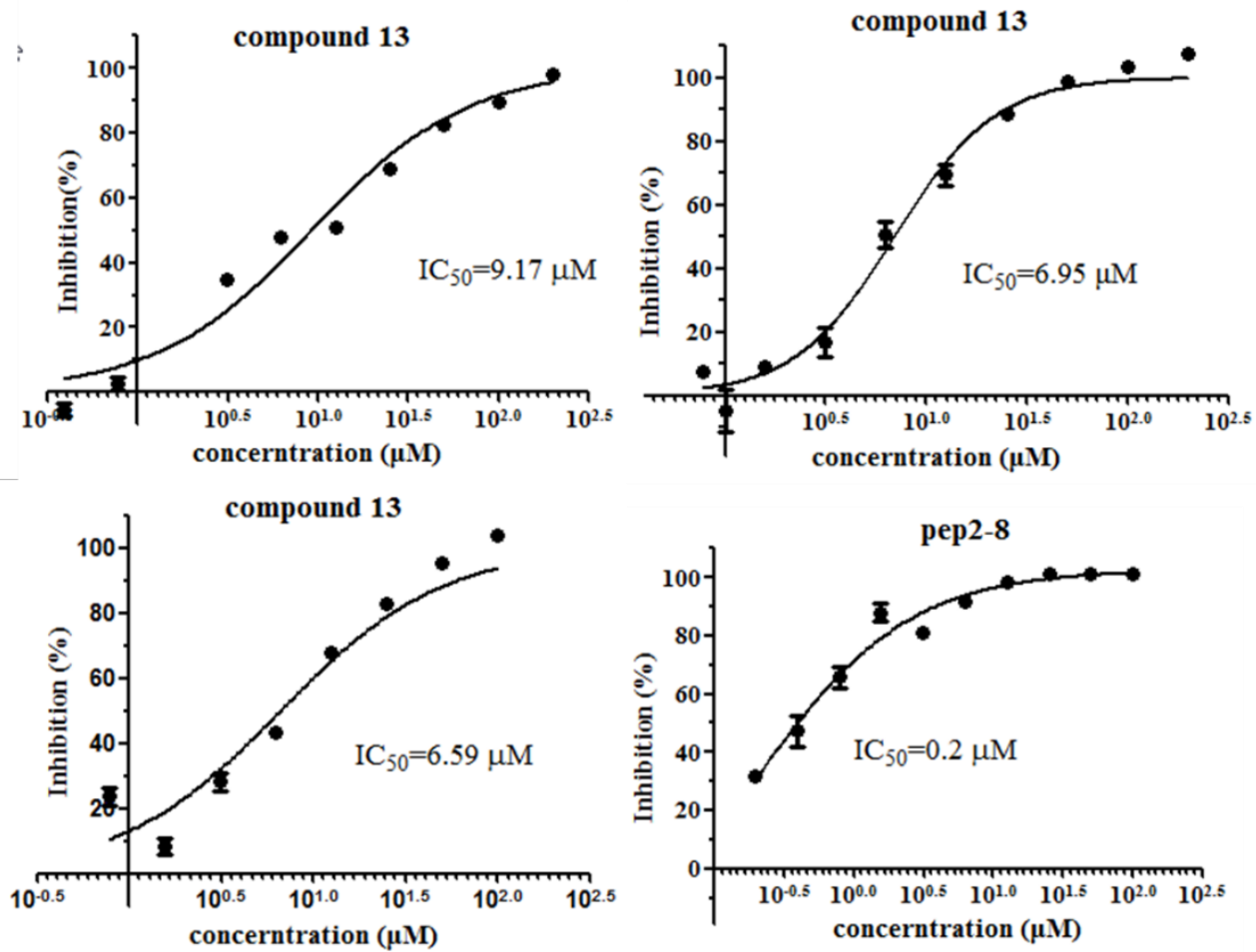

Figure S1. The inhibitory activities of $\mathbf{1 3}$ and pep2-8 against PCSK9-LDLR binding determined by duplicated ELISA assays. 


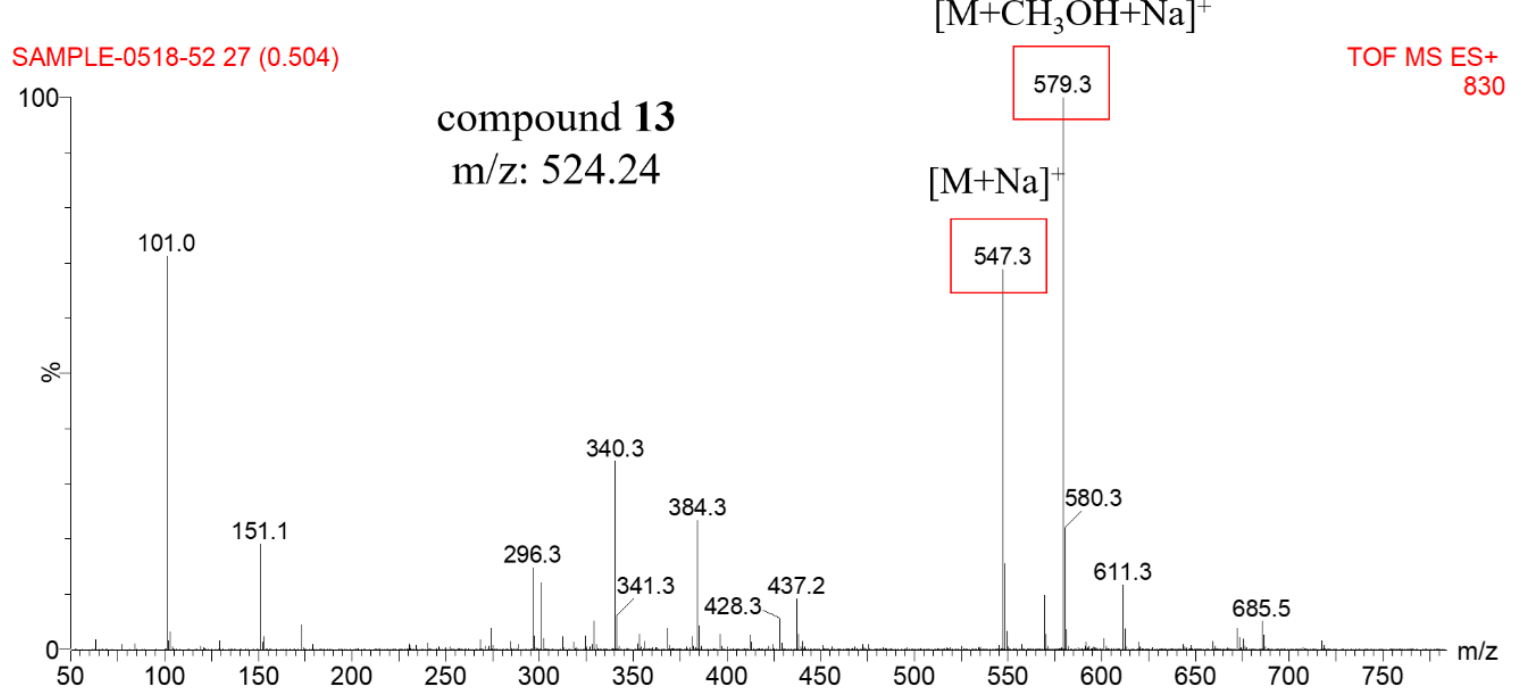

Figure S2. The mass spectrometry analysis of compound $\mathbf{1 3 .}$ 

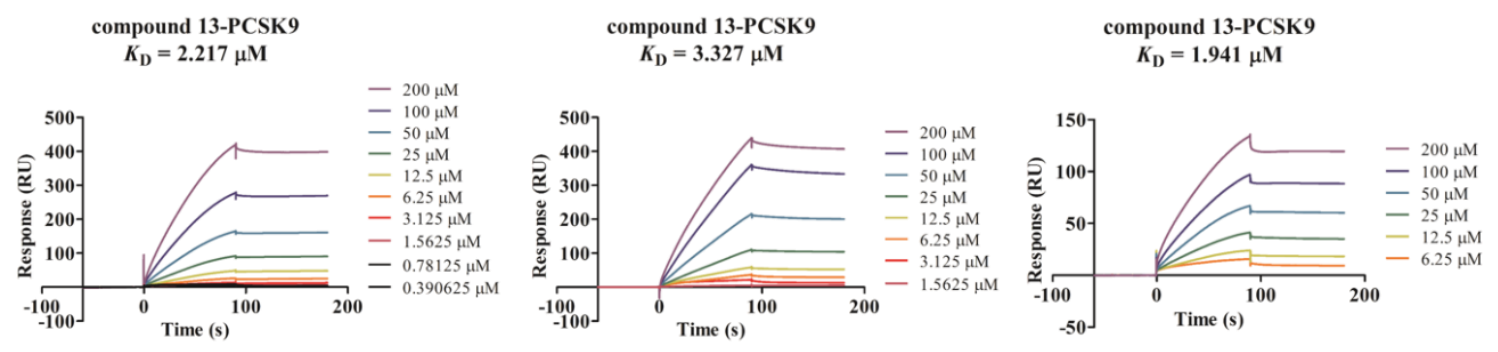

Figure S3. The $K_{\mathrm{D}}$ values of $\mathbf{1 3}$ were obtained from three independent SPR tests. 


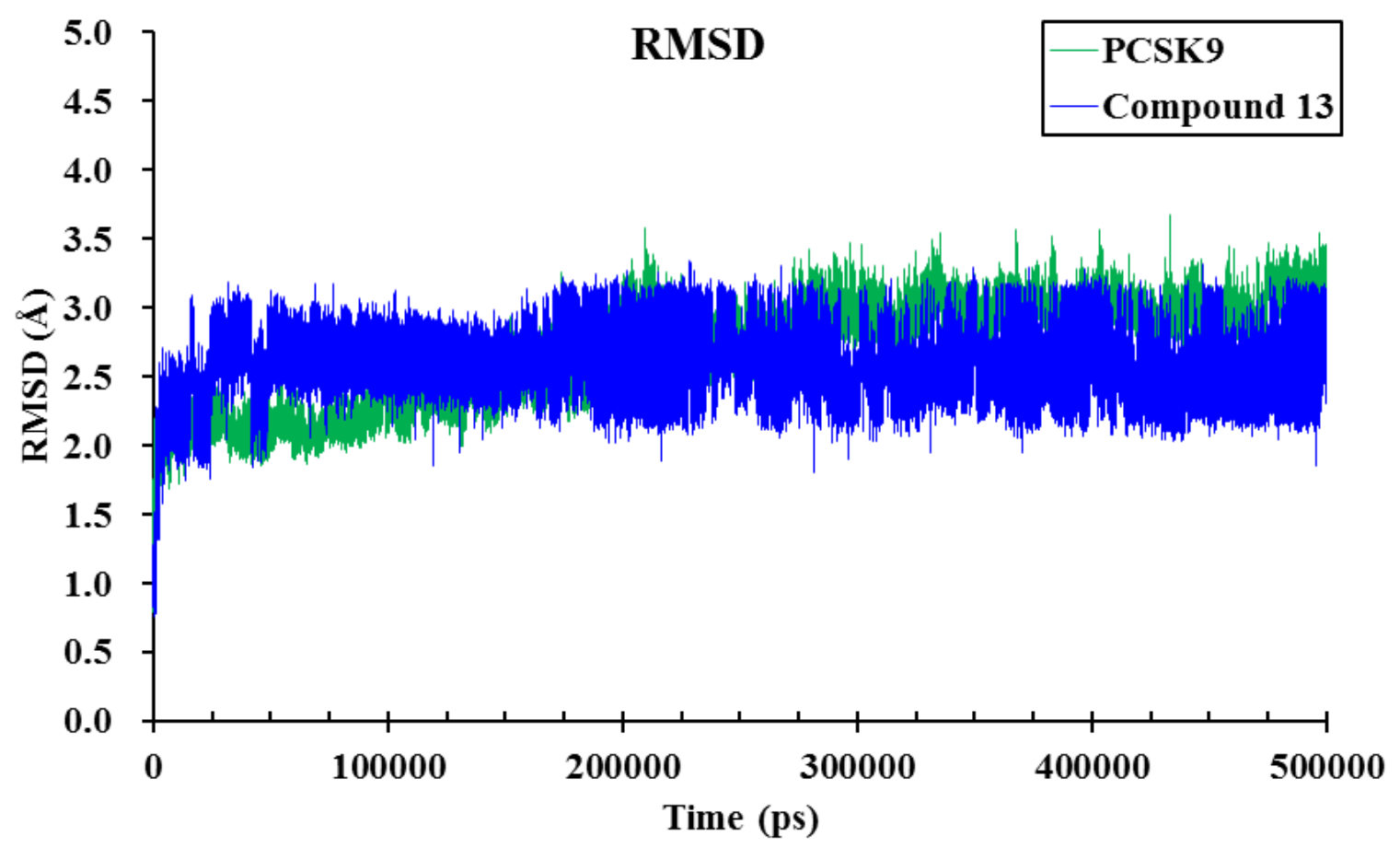

Figure S4. The RMSD values of the protein-ligand complex of PCSK9 and $\mathbf{1 3}$ in the entire molecular dynamics simulation of $500 \mathrm{~ns}$. 


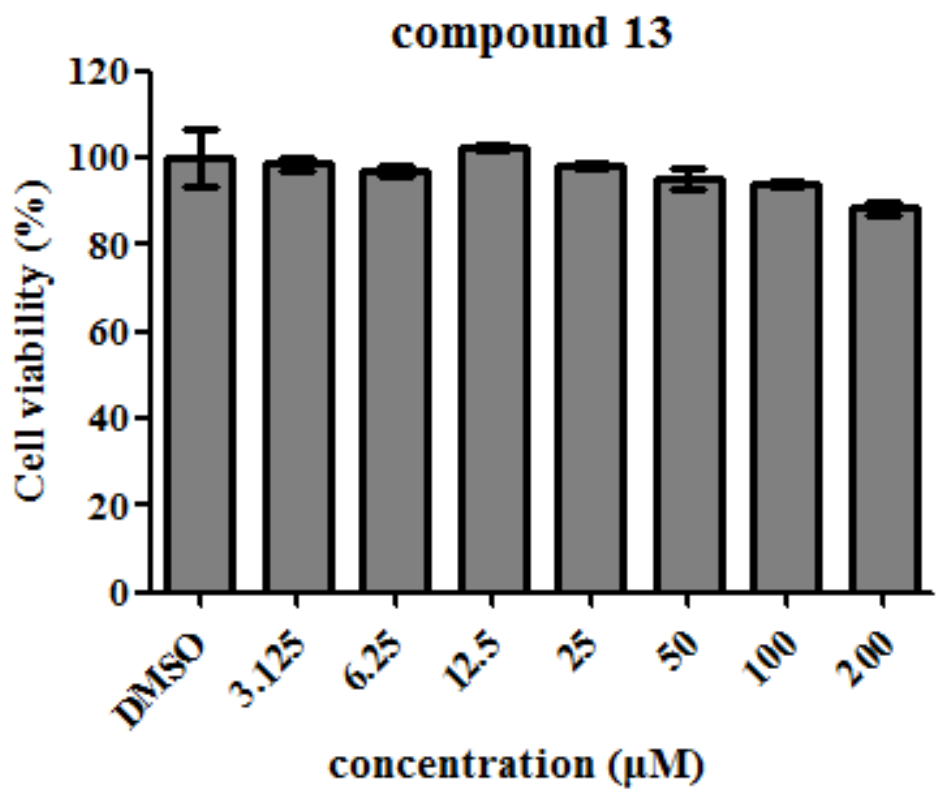

Figure S5. The effect of $\mathbf{1 3}$ on cell viability was determined with MTT assay. 